\title{
97. A Study of Cerebral Edema by Ultrasonic
}

\author{
Yutaka InABa, Hisao Maruta and Hiroshi Hata \\ 2nd Surgical Division, Tokyo Medical and Dental College
}

We have measured the ultrasonic attenuation of the brain which is affected by head-injuries or craniotomy by the reflection method using the ultrasonic diagnostic apparatus of J.R.C. Ltd. In case of head-injuries and craniotomy we have found conspicuous increasing tendency of the ultrasonic attenuation.

Comparing of the midline echo shift with the ultrasonic attenuation, we have noticed that the grade of the ultrasonic attenuation has been parallel to that of the cerebral edema in the cases of the operations of unilateral hemisphere. In the certain cases we have discovered the fact that we can recognize the grade of the cerebral edema by measuring of the midline echo shift.

We would like to present here how to measure the ultrasonic attenuation and the midline echo shift, and their reliability. We would also report the data from the three groups of study; (A) craniotomy (extradural procedure), (B) craniotomy (intradural procedure), and (C) control.

We have considered the relationship among the data, clinical symptoms, cerebrospinal fluid pressure and electro-encephalography.

\section{Study on the Dynamic Test of Traumatic Brains by Measuring the Ultrasonic Loss

\author{
Masuhisa OKa, Yasushi KuJiRaoka, Yasuaki NaIto, \\ and Hitoshi SaKamoto \\ Dept. of Surgery, Osaka Labor-Disease and Welfare Hospital
}

Ultrasound attenuates gradually in living tissues during its propagation. The ultrasonic loss is dependent on the tissue density, that of the same tissue fluctuating under various conditions. Changes in the content of body water may play a role on the cause of the ultrasonic loss. Patients suffering from head injuries and/or received angiographies and cerebral operations have been examined by continuous measuring of ultrasonic loss which was found useful for detecting some edematous states of traumatic brains. Up-to-date fifteen cases of brain tumor operations and head injuries were tested following surgical procedures by the ultrasonic diagnostic unit combined with an attenuator. A decrease of the ultrasonic attenuation or an increase of the intracranial fluid is observed about ten 